Copyright 2016 Society of Photo-Optical Instrumentation Engineers. One print or electronic copy may be made for personal use only. Systematic reproduction and distribution, duplication of any material in this paper for a fee or for commerical purposes, or modification of the content of the paper are prohibited.

\title{
LEO-ground scintillation measurements with the Optical Ground Station Oberpfaffenhofen and SOTA/OPALS space terminals
}

\author{
Florian Moll*a, Dimitar Kolev ${ }^{\mathrm{b}}$, Matthew Abrahamson ${ }^{\mathrm{c}}$, Christopher Schmidt ${ }^{\mathrm{a}}$, Ramon Mata Calvo ${ }^{\mathrm{a}}$, \\ Christian Fuchs ${ }^{\mathrm{a}}$ \\ ${ }^{a}$ German Aerospace Center, Oberpfaffenhofen, Muenchener Strasse 20, 82234 Wessling; ${ }^{b}$ National \\ Institute of Information and Communications Technology, Nukui-Kitamachi 4-2-1, Tokyo, Koganei; \\ ${ }^{\mathrm{c} J} J$ et Propulsion Laboratory, 4800 Oak Grove Drive, M/S 161-135, Pasadena, CA 91109
}

\begin{abstract}
The optical satellite-ground channel is turbulent and causes scintillation of the power received by a ground based telescope. Measurements are important to quantify the effect and evaluate common theory. A telescope with $40 \mathrm{~cm}$ primary mirror is used to measure the signals from the OPALS terminal on the International Space Station and the SOTA terminal on the SOCRATES satellite. The measurement instrument is a pupil camera from which images are recorded and intensity scintillation index, power scintillation index, probability density function of intensity and intensity correlation width are derived. A preliminary analysis of measurements from three satellite passed is performed, presented and discussed. The intensity scintillation index ranges from $\sim 0.25$ to $\sim 0.03$ within elevations of 26 to $66 \mathrm{deg}$. Power scintillation index varies from $\sim 0.08$ to $\sim 0.006$ and correlation width of intensity between $\sim 11$ and $\sim 3 \mathrm{~cm}$. The measurements can be used to estimate the fluctuation dynamics to be expected for a future operational ground receiver. The measurements are compared to model calculations based on the $\mathrm{HV}_{5 / 7}$-profile. Good agreement is observed to some part in the intensity scintillation index. Agreement is less for the power scintillation index and intensity correlation width. The reason seems to be a reduction of aperture averaging in some sections of the measurements due to increased speckle size. Finally, topics for future work are identified to improve the measurement analysis and deeper investigate the origin of the observed behavior.
\end{abstract}

Keywords: Satellite communications, free-space optical communications, scintillation, low earth orbit, Optical Ground Station Oberpfaffenhofen, SOTA, OPALS

\section{INTRODUCTION}

Optical wireless communications are an emerging technology and can be applied in scale sizes ranging from inter-chip connections to indoor, outdoor and space links [1]. Applications in the space domain are links between ground, LEO, GEO and deep space. In the LEO-GEO scenario, first commercial systems start operations like the European Data Relay System which employs an optical inter-satellite connection to relay data from Low Earth Orbit (LEO) satellite over a geostationary satellite to ground [2], [3]. The LEO-ground channel is in particular very interesting for different applications like the connection of LEO constellations to the ground network and data retrieval from Earth observation satellites. The first laser downlink system transmitting operational data will probably be the OSIRIS communications payload onboard DLR's BIROS satellite [4] which is in orbit since 22nd June 2016. The LEO-ground channel is complex since link distance, as well as the atmospheric path between transmitter and receiver and the slew rate, change during the contact time. Only few LEO satellite transmitters were available for experiments so far like the LUCE terminal on OICETS (Optical Interorbit Communications Engineering Test Satellite) [5], the LCT (Laser Communications Terminal) on the TerraSAR and NFIRE (Near Field Infrared Experiment) satellites [6], the OPALS (Optical Payload for Lasercomm Science) terminal on the International Space Station (ISS) [7] and the SOTA (Small Optical TrAnsponder) terminal on SOCRATES (Space Optical Communication Research Advanced Technology Satellite) [8]. Thus only few LEO-ground laser signal measurements are available till now which are the measurements with ground stations in Japan, USA, France and Germany. The majority of the published scintillation measurements used a wavelength of $847 \mathrm{~nm}$ and $1064 \mathrm{~nm}$ and were recorded with OICETS and the NFIRE satellite as space sources. Since nowadays most LEO transmitters deploy, or plan to, a transmission wavelength in the spectrum around $1550 \mathrm{~nm}$ for direct to earth links, channel characterization and measurements are in particular interesting with this wavelength which is used by the OPALS and SOTA terminals. Measurements with these sources and preliminary analysis are discussed in

(C) 2016 SPIE $\cdot$ CCC code: $0277-786 X / 16 / \$ 18 \cdot$ doi: $10.1117 / 12.2254809$ 
[9], [8], and [10]. In [9], measurements are discussed which were taken with the SOTA terminal and the MeO ground station at Caussols, France. Apertures of $1.5 \mathrm{~m}, 0.4 \mathrm{~m}$ and $0.2 \mathrm{~m}$ were alternatingly used to measure the received power at $1549 \mathrm{~nm}$ within three passes. In [8], emphasis is on link budget design, measurement of received optical power and evaluation of error correction code performance. The transmitter is the same source, but the receiver is a ground station in Koganai, Japan. In [10], the focus is on testing an adaptive optics system. The source is the OPALS terminal on the ISS, the receiver is a ground station in Wrightwood, California. Apparently, no detailed measurement analysis is published so far that shows the intensity and power scintillation in dependence on elevation for a transmission wavelength in the spectrum around $1550 \mathrm{~nm}$. We present a preliminary analysis of measurements here in order to contribute to a later channel model development.

We performed measurements of incident intensity at the Optical Ground Station Oberpfaffenhofen (OGS-OP) near Munich, Germany. The space sources are the SOTA terminal onboard SOCRATES and the OPALS terminals onboard the ISS. The measurements are analyzed and intensity and power scintillation index over link elevation is given as well as the intensity correlation width.

\section{THEORETICAL BACKGROUND}

Atmospheric turbulence distorts the wave-front of the propagating wave. This results in scintillation of irradiance at the receiver telescope plane and scintillation of power at the receiver front-end plane. The scintillation of irradiance is quantified by the irradiance scintillation index $\sigma_{\mathrm{I}}^{2}[-]$. For the case of a satellite downlink, this term can be modelled over elevation by

$$
\sigma_{\mathrm{I}}^{2}=\exp \left[\frac{0.49 \sigma_{\mathrm{R}}^{2}}{\left(1+1.11 \sigma_{\mathrm{R}}^{12 / 5}\right)^{7 / 6}}+\frac{0.51 \sigma_{\mathrm{R}}^{2}}{\left(1+0.69 \sigma_{\mathrm{R}}^{12 / 5}\right)^{5 / 6}}\right]-1
$$

which is valid for weak, moderate and strong irradiance fluctuations [11]. The Rytov index $\sigma_{\mathrm{R}}{ }^{2}[-]$ is given by

$$
\sigma_{\mathrm{R}}^{2}=2.25 k^{7 / 6} \sec ^{11 / 6}(90-\varepsilon) \int_{h_{0}}^{H} C_{\mathrm{n}}^{2}(h)\left(h-h_{0}\right)^{5 / 6} d z .
$$

with the wave number $k\left[\mathrm{~m}^{-1}\right]$, transmitter height $H[\mathrm{~m}]$, receiver height $h_{0}[\mathrm{~m}]$, link elevation $\varepsilon$ [deg] and the index of refraction structure constant profile $C_{\mathrm{n}}^{2}(\mathrm{~h})\left[\mathrm{m}^{-2 / 3}\right]$. Receiver height and transmitter height are chosen to be $1 \mathrm{~m}$ and $20 \mathrm{~km}$. The second value is chosen since no relevant impact of turbulence is expected beyond $20 \mathrm{~km}$ height.

The scintillation of received power is quantified by the power scintillation index $\sigma_{\mathrm{P}}^{2}[-]$. For the case of a satellite downlink this is modelled over elevation angle for the case of weak irradiance fluctuations by

$$
\sigma_{P}^{2}=8.7 k^{7 / 6}\left(H-h_{0}\right)^{5 / 6} \sec ^{11 / 6}\left(90^{\circ}-\varepsilon\right) \times \operatorname{Re} \int_{h_{0}}^{H} C_{n}^{2}(h)\left[\left(\frac{k D^{2}}{16 L}+i \frac{h-h_{0}}{H-h_{0}}\right)^{5 / 6}-\left(\frac{k D^{2}}{16 L}\right)^{5 / 6}\right] d h
$$

[11] with the telescope aperture diameter $D[\mathrm{~m}]$ and the length of the propagation path $L[\mathrm{~m}]$. The $C_{\mathrm{n}}{ }^{2}$-profile is modelled by the Hufnagel-Valley profile

$$
C_{\mathrm{n}}^{2}(h)=0.00594 \cdot\left(v_{\mathrm{rms}} / 27\right)^{2} \cdot\left(10^{-5} \cdot h\right)^{10} \cdot e^{-h / 1000}+2.7 \cdot 10^{-16} \cdot e^{-h / 1500}+A \cdot e^{-h / 100} .
$$

The parameterization is via the root mean squared wind speed $v_{\mathrm{rms}}[\mathrm{m} / \mathrm{s}]$, between $5 \mathrm{~km}$ and $20 \mathrm{~km}$ height, and a $C_{\mathrm{n}}^{2}$ ground value $A\left[\mathrm{~m}^{-2 / 3}\right]$. A special case is the parameterization with $v_{\mathrm{rms}}=21 \mathrm{~m} / \mathrm{s}$ and $A=1.7 \mathrm{e}^{-14} \mathrm{~m}^{-2 / 3}$ which results in the $\mathrm{HV}_{5 / 7}$-profile since it produces an atmospheric coherence diameter of $5 \mathrm{~cm}$ and an isoplanatic angle of $7 \mu \mathrm{rad}$ at a wavelength of $0.5 \mu \mathrm{m}$ [11]. These values are often assumed by astronomers for nighttime conditions. However, the Hufnagel-Valley model is often not suitable for daytime conditions [12].

The correlation width of intensity is derived from the normalized covariance function of intensity as its 1/e-point. A simple model for the case of weak fluctuation conditions is given by 
$\rho_{\mathrm{c}}=\sqrt{\frac{45 \cdot 10^{3} \cdot \sec \left(90^{\circ}-\varepsilon\right)}{k}}$

for $\sigma_{\mathbb{R}}^{2}<<1$ [13], which fits, with some limitations, to the aforementioned conditions.

\section{MEASUREMENT SETUP}

\subsection{General setup}

The receiver station is the Optical Ground Station Oberpfaffenhofen (OGS-OP) near Munich, Germany. The space sources are the OPALS terminal on the International Space Station and the SOTA terminal on SOCRATES. The ground station sought link acquisition by using beacon lasers which were attached to the same telescope or the neighboring TOGS.

\subsection{Ground station}

The functional block diagram of the ground station is shown in Figure 1 (left). Link acquisition is performed and optical tracking guarantees link lock between the satellite and the ground station. The laser light is received with the telescope and guided to the measurement devices using collimation and focusing optics. The measurement devices are a PIN receiver and a pupil camera. The PIN receiver is a modified Thorlabs PDA 400. The signal is sampled with a NI PCI6259 DAQ (16 bit, $20 \mathrm{ksamples} / \mathrm{s}$ ). The pupil camera comprises a pupil imaging system behind the telescope and a Xenics Xeva Trigger IR camera free running at $100 \mathrm{fps}$. An image of the ground station is given in Figure 1 (right). The clamshell dome hosts the $40 \mathrm{~cm}$ Cassegrain telescope which is mounted on a custom made aluminum fork mount.
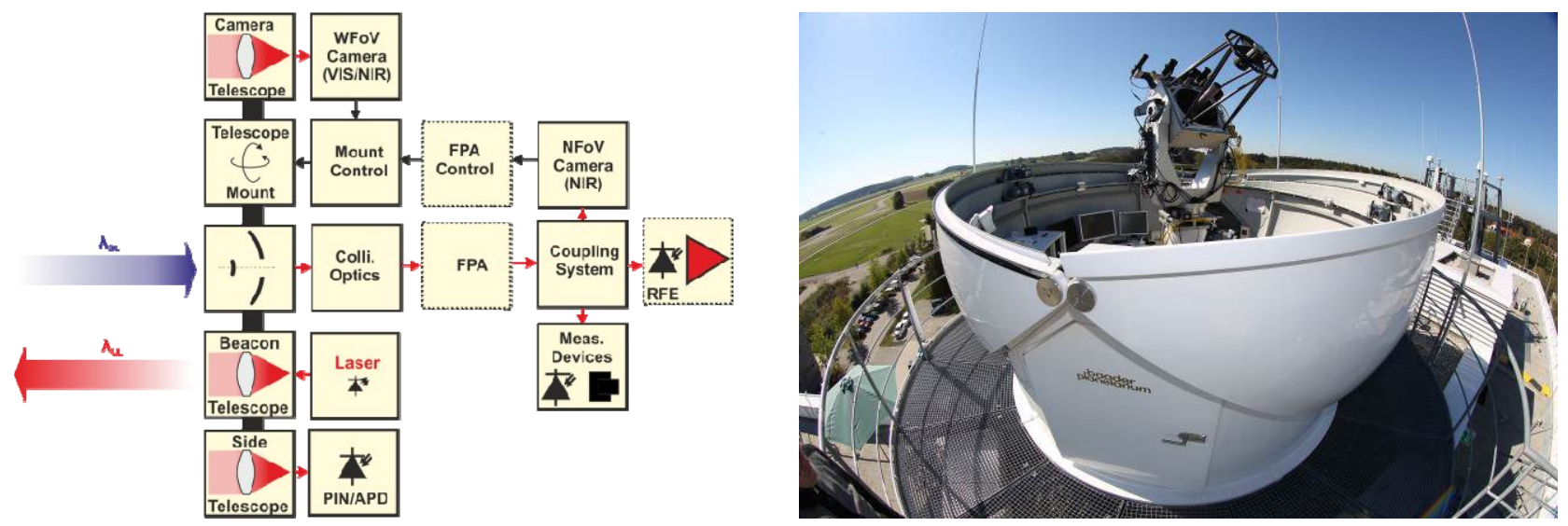

Figure 1. Functional block diagram of measurement setup (left) and image of Optical Ground Station Oberpfaffenhofen (right). source: DLR.

\subsection{Space source 1: OPALS}

The OPALS payload was developed by NASA-JPL for demonstration of space-to-ground optical communications links aboard the International Space Station (ISS) [7]. OPALS was launched in April 2014 and installed on Express Logistics Carrier-1 (ELC-1) in May 2014. The first successful experiment was conducted in June 2014 to a ground station located in Wrightwood, California, USA. Measurements of several experiments for validation of up- and downlink were also presented [14]. Afterwards, OPALS was also used for international experiments with partners at DLR, ESA and CNES. OPALS is designed for compatibility with an unmodulated $967 \mathrm{~nm}$ ground-based beacon system to enable precise pointing of the downlink beam. The sensitivity of OPALS made the use of a beacon with a rather large divergence angle of about $1.7 \mathrm{mrad}$ possible, allowing for relatively easy pointing constraints during the 25-deg elevation acquisition at the beginning of the experiment. DLR's ground station design made use of two laser sources in transmitter diversity configuration with up to $7 \mathrm{~W}$ each. Furthermore, the communication system of OPALS uses $1550 \mathrm{~nm}$ with On-OffKeying modulation. The maximum data rate is $50 \mathrm{Mbps}$ and a Reed-Solomon code is used for error correction purposes. 
This enables the transmission of e.g. high-resolution video data. DLR focused on the gathering of atmospheric measurement data, as can be seen later in the text.

\subsection{Space source 2: SOTA}

SOTA has been developed by National Institute of Information and Communications Technology (NICT), Japan and is the payload of a 50-kg class microsatellite called SOCRATES [15]. Full mission success has been achieved in June 2015 and international experiment campaign between SOTA (NICT) and the optical ground stations (OGS) of different partners has started. A scheme of SOTA is shown in Figure 2 and the main SOTA parameters are listed in Table 1 and Table 2. The acquisition and tracking sensor in the image represents the coarse tracking sensor. The fine tracking sensor is installed behind the optical antenna for Tx4 and Rx. Tx1 is next to the coarse tracking sensor. From this scheme it is clear that Tx1 links have only coarse tracking and the fine tracking and acquisition is available only for Tx4 links. This explains the significant difference in the divergence angle between both laser sources since in the case of Tx1 it is harder to align the laser link. The uplink beacon must emit at $1064 \mathrm{~nm}$ wavelength so that SOTA sensors can detect it. It is furthermore necessary for the beacon to hit SOTA for up to several seconds while the gimbal slowly adjusts and the optical link is established. Careful calculation of the beacon divergence angle, considering the minimum required irradiance on SOTA (Table 2) side and the orbit uncertainty is necessary.

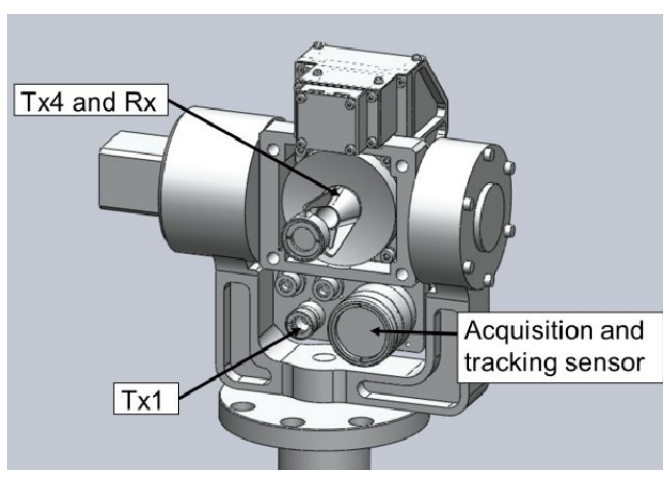

Figure 2. A scheme of the transmitters and coarse tracking sensor positions.

Table 1. SOTA downlink characteristics.

\begin{tabular}{|c|c|c|c|}
\hline Issues & Tx1 & $\mathrm{Tx} 4$ & Others \\
\hline Wavelength (nm) & 976 & 1549 & at $25^{\circ} \mathrm{C}, 0.1 \mathrm{~nm} / \mathrm{deg}$ \\
\hline Polarization & Random & RHCP & \\
\hline Data rate $(\mathrm{Mbps})$ & 10 or 1 & 10 or 1 & selectable \\
\hline Intensity (MW/sr) & 0.89 & 0.57 & \\
\hline Divergence angle $(\mu \mathrm{rad})$ & 500 & 223 & $-3 \mathrm{~dB}$ Full width \\
\hline Pointing loss (dB) & -3.4 & -5.7 & \\
\hline Atmospheric loss (dB) & -5.6 & -3.9 & at NICT OGS \\
\hline Irradiance $\left(\mathrm{nW} / \mathrm{m}^{2}\right)$ & 112 & 63 & $\begin{array}{l}\text { at ground, } 1000 \mathrm{~km} \\
\text { distance }\end{array}$ \\
\hline Modulation & OOK & OOK & $\begin{array}{l}\text { NRZ, PRBS- } 15 \text {, the } \\
\text { generating polynomial is } \\
X^{\wedge} 15+X^{\wedge} 14+1 .\end{array}$ \\
\hline
\end{tabular}

Table 2. SOTA uplink (tracking and acquisition) characteristics.

\begin{tabular}{|l|l|l|}
\hline Issues & $\begin{array}{l}\text { Acquisition and } \\
\text { coarse tracking }\end{array}$ & Fine tracking \\
\hline Wavelength $(\mathrm{nm})$ & $1064 \pm 3$ & $1064 \pm 3$ \\
\hline Required irradiance $\left(\mu \mathrm{W} / \mathrm{m}^{2}\right)$ & $11 \sim 209$ & $11 \sim 209$ \\
\hline
\end{tabular}




\section{MEASUREMENT CAMPAIGN}

The measurement results are derived from three satellite passes in 2015 and 2016. In three cases, weather and other parameters enabled successful link acquisition and performing atmospheric measurements. For the OPALS campaign, two beacon lasers systems with $976 \mathrm{~nm}$ were used whereas two $1064 \mathrm{~nm}$ systems were deployed to provide the beacon signal for the SOTA payload. Two transmitter telescopes were installed aside the ground stations primary mirror. The selected satellite passes for all three experiments provide different characteristics in terms of measurement time, maximum elevation and thus atmospheric influences on the signal. Table 3 gives the characteristics of the selected downlink experiments for OPALS and SOTA.

Table 3. Characteristics of satellite passes.

\begin{tabular}{|l|l|l|l|l|l|l|}
\hline Date & Time [UTC] & Sunset [UTC] & Exp. designation & Max. el [deg] & Duration of pass [s] & Duration of measurement [s] \\
\hline 12.10 .2015 & $16: 37: 49$ & $16: 33$ & OP12-10-15 & 74 & 607 & 69 \\
\hline 06.06 .2016 & $20: 52: 35$ & $19: 11$ & OP06-06-16 & 66 & 607 & 142 \\
\hline 06.05 .2016 & $22: 50: 01$ & $18: 35$ & SO06-05-16 & 58 & 750 & 84 \\
\hline
\end{tabular}

\section{RESULTS AND DISCUSSION}

Signal recordings of the pupil camera are analyzed by using the analysis method of the intensity correlation width described in [16]. However, 100 images are used as sliding window. Intensity and power scintillation index are also determined using the camera images and directly calculated from the pixel values.

The measurement results are shown in the following. For the sake of comparison, model calculations are given which are based on the $\mathrm{HV}_{5 / 7}$-profile. Three measurements are shown here, one with SOTA (SO06-05-16) and two with OPALS (OP06-06-16, OP12-10-15) which were all during or beginning of nighttime (compare Table 3). The measured intensity scintillation index over elevation is depicted in Figure 3 (left). The dashed black line and the solid black line denote $\sigma_{I}^{2}$ and $\sigma_{\mathrm{R}}{ }^{2}$ as calculated with equation 1 and 2 . The red, blue and green lines denote the measurements. The red and green lines (SO06-05-16 and OP12-10-15) fit quite well to the theoretical curves showing some dynamics. OP12-10-15 and OP06-06-16 show measurements in ascending and descending elevation of the pass which is seen as a second line with same color, respectively. OP06-06-16 appears to be well below the prediction of the $\mathrm{HV}_{5 / 7}$-profile. The probability density distributions at $33 \mathrm{deg}$ (solid black) and $54 \mathrm{deg}$ (solid blue) elevation of SO06-05-16 are shown in Figure 3 (right). They reveal the expected lognormal shape. These measured distributions are averages of several hundred pupil images to obtain a representative for the chosen elevations. The dashed curves show the theoretical shape for a given intensity scintillation index.
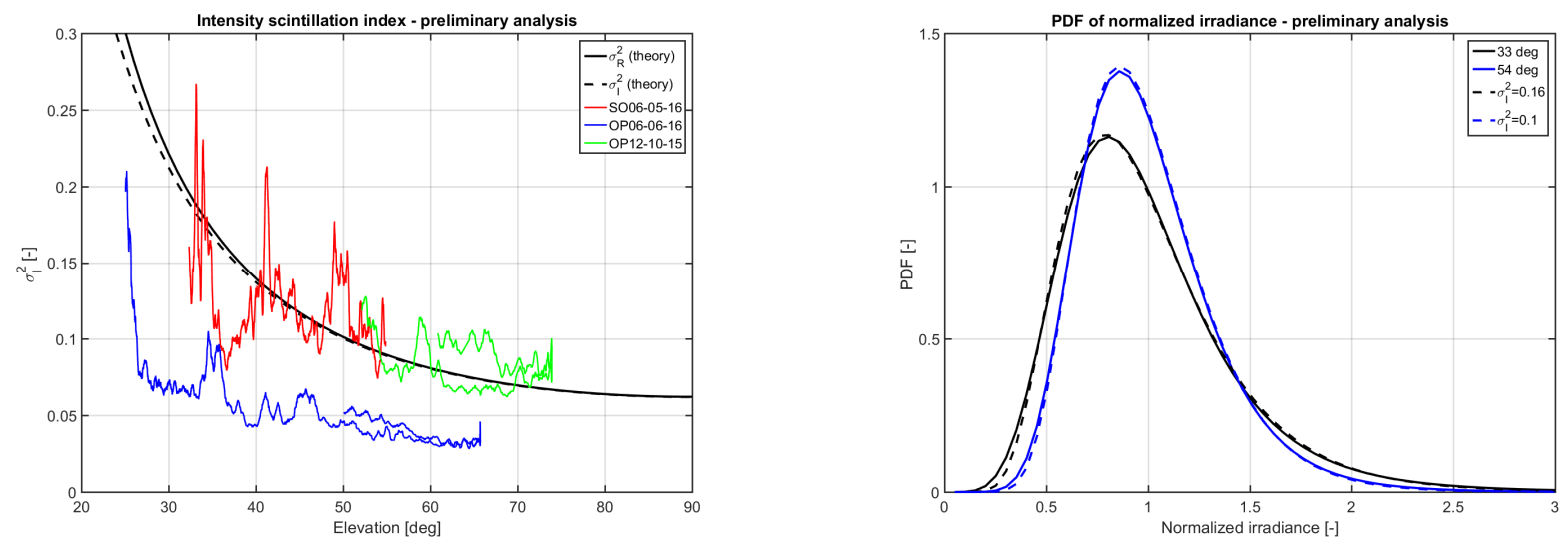

Figure 3. Measured and theoretical intensity scintillation index (left) and probability density function of two selected elevations from the SO06-05-16 measurement.

The power scintillation index is shown in Figure 4 (left). The SOTA SO06-05-16 measurement fits reasonably well with the $40 \mathrm{~cm}$ prediction of the $\mathrm{HV}_{5 / 7}$. However, no clear change with elevation is observed. Parts of the OPALS OP06-0616 measurement (below $29 \mathrm{deg}$ and between 33 and $46 \mathrm{deg}$ ) also fit reasonably well being slightly over the $\mathrm{HV}_{5 / 7}$ prediction. However, the other intervals of the measurement (from 30 to $33 \mathrm{deg}$ and above $46 \mathrm{deg}$ ) are far over the 
prediction. Also the OPALS OP12-10-15 measurement reveals higher values even increasing with elevation. Similar behavior is observed in the analysis of the intensity correlation width. The SOTA SO06-05-16 measurement behaves quite much as expected, i.e. decreasing, slightly, with elevation, however, being well below the prediction of the model given in equation 5. Correlation width of the OPALS OP06-06-16 is generally higher with sharp increase from 30 to 33 deg and above $46 \mathrm{deg}$. This is correlated to the behavior of the power scintillation index (compare left Figure). It must be noted that interpretation of this behavior should only be done qualitatively here due to the finite measurement aperture of $40 \mathrm{~cm}$. This limits the measurement span to an upper bound of $<40 \mathrm{~cm}$. The OPALS OP12-10-15 measurement reveals a smaller correlation width which even slightly increases with elevation.

The cause for the difference in the measurement results amongst the individual satellite passes are the time of the day, different macro weather situations and link azimuth angle. The quantitative difference in intensity scintillation index can be explained by different time of the day and the macro weather situations. This is quite usual for atmospheric conditions for different dates and times. Higher complexity is discovered by observation of the power scintillation index and intensity correlation width. The second reveals large and sudden changes in the intensity pattern, i.e. the speckle size increases. This in turn increases the power scintillation index since aperture averaging decreases. This sudden change is likely be caused by the changing azimuth viewing direction during the pass. Indeed, the ground station is located with different landscape around. Agricultural fields, forest and lakes dominate the southern direction whereas northern direction shows more urban population. The observed strong irregularities in power scintillation index and correlation width happen in northern viewing direction. Apparently, the turbulence in this direction had a rather low intensity scintillation index, meaning smaller $\mathrm{C}_{\mathrm{n}}^{2}$ values along the path. The sudden change in intensity correlation width without parallel change in intensity scintillation index seems to be caused by a change in the turbulence spectral shape, possibly non-Kolmogorov while the southern turbulence spectrum is likely more Kolmogorov.
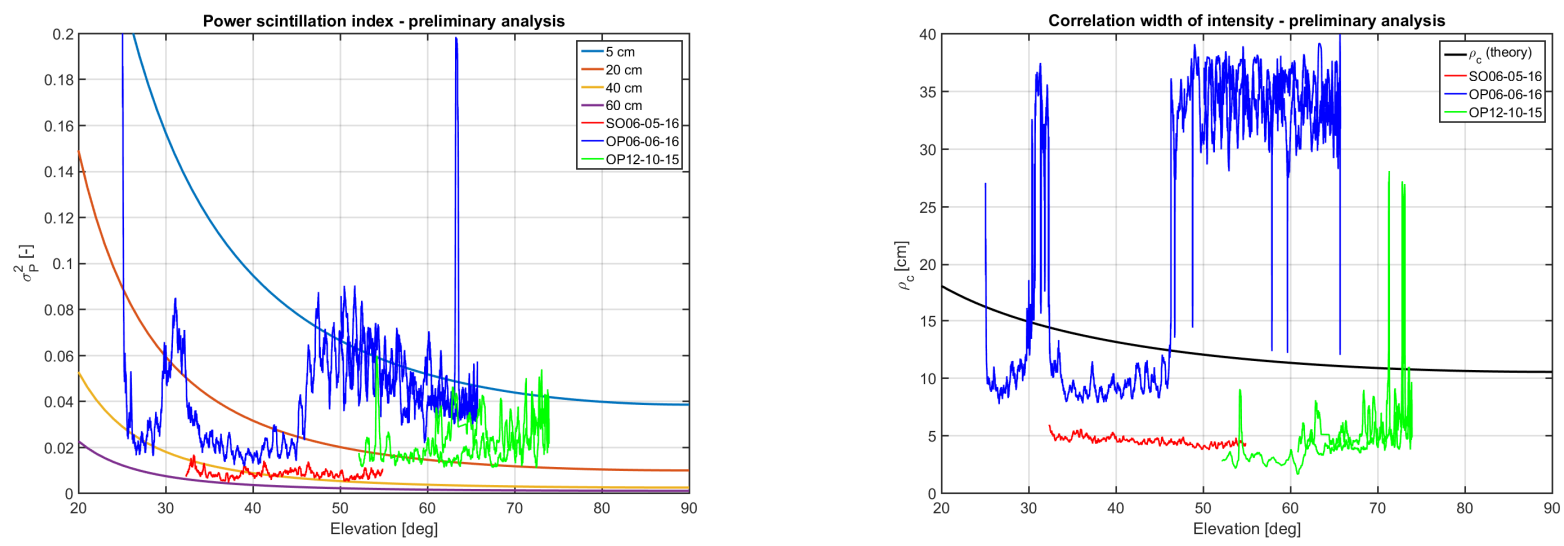

Figure 4. Measured and theoretical power scintillation index (left) and intensity correlation width (right).

\section{SUMMARY}

We presented the preliminary analysis of scintillation measurements with the Optical Ground Station Oberpfaffenhofen and OPALS and SOTA as space laser sources. The measurement method is the analysis of pupil camera recordings in which we derived the intensity scintillation index, power scintillation index, probability density function of intensity and correlation width of intensity. The intensity scintillation index shows expected behavior, i.e. decreases with increasing elevation angle. Some does the intensity PDF. The power scintillation index and intensity correlation width show unexpected behavior in one satellite pass. This is assumed to be caused by different landscape structure for different azimuthal angles around the site location.

Several topics were identified which need deeper analysis. First of all, the unexpected behavior of power scintillation index and intensity correlation width, i.e. the sudden and strong deviations from the expected run in trial OP06-06-16 must be further analyzed. The effect of different overflight times, azimuth view and metro scale weather must be investigated. Furthermore, noise impact of background light must be taken into consideration as error source. The $\mathrm{HV}_{5 / 7^{-}}$ profile works fine for the sake of a first comparison, but a better suited $\mathrm{C}_{\mathrm{n}}{ }^{2}$-profile should be chosen for a more accurate theoretical analysis. The same is valid for the model of intensity correlation width. The model in this paper has been 
chosen for a first iteration. A more accurate model which also takes the in-situ $\mathrm{C}_{\mathrm{n}}^{2}$-profile into account should be used. Finally, the analysis method shall be optimized to erase remaining artefacts and validated with a second measurement setup.

\section{ACKNOWLEDGEMENTS}

The SOTA measurements were obtained in a collaborative work of the German Aerospace Center (DLR) Institute of Communications and Navigation and the National Institute of Information and Communications Technology. The OPALS measurements were obtained in a collaborative work of the German Aerospace Center (DLR) Institute of Communications and Navigation and Jet Propulsion Laboratory (JPL) of NASA.

The authors would like to thank everyone who was involved in the measurement campaign and the preparation. Special thanks go to NICT and JPL who built and operated the space terminals and kindly offered them for this great international cooperation.

\section{REFERENCES}

[1] M. A. Khalighi and M. Uysal, "Survey on Free Space Optical Communication: A Communication Theory Perspective," vol. 16, no. 4, pp. 2231-2258, 2014.

[2] H. Hauschildt, F. Garat, H. Greus, K. Kably, J.-P. Lejault, H. L. Moeller, A. Murrell, J. Perdigues, D. M. Witting, B. Theelen, M. Wiegand, and A. Hegyi, "European Data Relay System - one year to go!," in Proc. International Conference on Space Optical Systems and Applications (ICSOS) 2014, 2014.

[3] H. Zech, F. Heine, D. Troendle, S. Seel, M. Motzigemba, R. Meyer, and S. Philipp-May, "LCT for EDRS: LEO to GEO Optical communications at 1,8 Gbps between Alphasat and Sentinel 1a," in Proc. of SPIE Vol. 9647, 2015, pp. 96470J-1-96470J-8.

[4] C. Schmidt, M. Brechtelsbauer, F. Rein, and C. Fuchs, "OSIRIS payload for DLR's BiROS satellite," in International Conference on Space Optical Systems and Applications 2014, 2014.

[5] Y. Fujiwara, M. Mokuno, T. Jono, T. Yamawaki, K. Arai, M. Toyoshima, H. Kunimori, Z. Sodnik, A. Bird, and B. Demelenne, "Optical inter-orbit communications engineering test satellite (OICETS)," Acta Astronautica, vol. 61, pp. 163-175, 2007.

[6] R. Fields, D. Kozlowski, H. Yura, R. Wong, J. Wicker, C. Lunde, M. Gregory, B. Wandernoth, and F. Heine, "5.625 Gbps Bidirectional Laser Communications Measurements Between the NFIRE Satellite and an Optical Ground Station," in Proc. of 2011 International Conference on Space Optical Systems and Applications, 2011, pp. 44-53.

[7] B. V. Oaida, M. J. Abrahamson, R. J. Witoff, J. N. B. Martinez, and D. A. Zayas, "OPALS: an optical communications technology demonstration from the International Space Station," in IEEE, 2013, pp. 1-20.

[8] H. Takenaka, Y. Koyama, D. Kolev, M. Akioka, N. Iwakiri, H. Kunimori, A. Carrasco-Casado, Y. Munemasa, E. Okamoto, and M. Toyoshima, "In-orbit verification of small optical transponder (SOTA) -Evaluation of satelliteto-ground laser communication links-," in Proc. of SPIE 9739, 2016, pp. 973903-1 - 973903-12.

[9] D.-H. Phung, E. Samain, N. Maurice, D. Albanesse, H. Mariey, M. Aimar, G. M. Lagarde, G. Artaud, J.-L. Issler, N. Vedrenne, M.-T. Vellut, M. Toyoshima, M. Akioka, D. Kolev, Y. Munemasa, H. Takenaka, and N. Iwakiri, "Telecom \& Scintillation first data analysis for DOMINO - Laser Communication between SOTA, onboard SOCRATES satellite, and MEO OGS," in Proceedings of ICSOS 2015, 2015.

[10] M. W. Wright, J. Kovalik, J. Morris, M. Abrahamson, and A. Biswas, "LEO-to-ground optical communications link using adaptive optics correction on the OPALS downlink," in Proc. of SPIE, 2016, pp. 973904-1 - 97390410.

[11] L. C. Andrews and R. L. Philips, Laser Beam Propagation through Random Media, 2nd ed. Belligham: SPIE Press, 2005.

[12] D. H. Tofsted, S. G. O'Brien, and G. T. Vaucher, "An atmospheric turbulence profile model for use in army wargaming applications I,” US Army Research Laboratory, 2006.

[13] L. C. Andrews, R. L. Phillips, and C. Y. Hopen, "Scintillation model for a satellite communication link at large zenith angles," SPIE Optical Engineering, vol. 39, pp. 3272-3280, 2000.

[14] A. Biswas, B. Oaida, K. S. Andrews, J. M. Kovalik, M. Abrahamson, and M. W. Wright, "Optical payload for lasercomm science (OPALS) link validation during operations from the ISS," in Proc. of SPIE9354, Free-Space Laser Communication and Atmospheric Propagation XXVII, 2015, p. 93540F. 
[15] Y. Koyama, M. Toyoshima, Y. Takayama, H. Takenaka, K. Shiratama, I. Mase, and O. Kawamoto, "SOTA: Small Optical Transponder for micro-satellite," in Proceedings of ICSOS 2011, 2011, pp. 97-101.

[16] F. Moll, "Experimental characterization of intensity scintillation in the LEO downlink," in 2015 4th International Workshop on Optical Wireless Communications (IWOW), 2015. 